\title{
Effect of block composition on thermal properties and melt viscosity of poly[2-(dimethylamino)ethyl methacrylate], poly(ethylene oxide) and poly(propylene oxide) block co-polymers
}

\author{
A. Vesterinen, S. Lipponen, J. Rich and J. Seppälä* \\ Aalto University School of Science and Technology, Polymer Technology Research Group, P.O. Box 16100, 00076 Aalto, \\ Finland
}

Received 23 December 2010; accepted in revised form 1 March 2011

\begin{abstract}
To modify the rheological properties of certain commercial polymers, a set of block copolymers were synthesized through oxyanionic polymerization of 2-(dimethylamino)ethyl methacrylate to the chain ends of commercial prepolymers, namely poly(ethylene oxide) (PEO), poly(ethylene oxide)-block-poly(propylene oxide)-block-poly(ethylene oxide) (PEO-PPO-PEO), and poly(propylene oxide) (PPO). The formed block copolymers were analysed with size exclusion chromatography and nuclear magnetic resonance spectroscopy in order to confirm block formation. Thermal characterization of the resulting polymers was done with differential scanning calorimetry. Thermal transition points were also confirmed with rotational rheometry, which was primarily used to measure melt strength properties of the resulting block co-polymers. It was observed that the synthesised poly[2-(dimethylamino)ethyl methacrylate]-block (PDM) affected slightly the thermal transition points of crystalline PEO-block but the influence was stronger on amorphous PPO-blocks. Frequency sweeps measured above the melting temperatures for the materials confirmed that the pre-polymers (PEO and PEO-PPO-PEO) behave as Newtonian fluids whereas polymers with a PDM block structure exhibit clear shear thinning behaviour. In addition, the PDM block increased the melt viscosity when compared with that one of the pre-polymer. As a final result, it became obvious that pre-polymers modified with PDM were in entangled form, in the melted state as well in the solidified form.
\end{abstract}

Keywords: rheology, PDM, DSC, block-copolymer

\section{Introduction}

Block co-polymers can have several properties that are not present in comparable statistical co-polymers, and interest in studying their properties has increased considerably during recent decade [1]. Block co-polymers can have, for example, the ability to form self-organized structures [2]. Especially co-polymers consisting of PDM (poly[2-(dimethylamino)ethyl methacrylate]), PEO (poly(ethylene oxide)), and/or PPO (poly(propylene oxide) blocks have found applications in the field of hydrophobiz- ing paper [3] or other hydrophilic surfaces [4], strengthening agents for wood fibre networks $[5,6]$ and even some biomedical applications [2]. In addition, some special solution properties have been found for copolymers containing cationizable PDM block. E.g. at high pH PEO-b-PDM will aggregate at the temperature where the PDM sequence is totally deprotonated and sufficiently hydrophobic [7]. PDM can also easily be modified into a permanently cationic form by methylation of the tertiary amine group to form a quaternary amine $[8,9]$.

\footnotetext{
${ }^{*}$ Corresponding author, e-mail: jukka.seppala@tkk.fi
}

(c) BME-PT 
One convenient way to polymerize 2-(dimethylamino)ethyl methacrylate, and via that route to synthesize block copolymers containing PDM segments, is to use potassium alcoholate $\mathrm{RO}^{-} \mathrm{K}^{+}$as an initiator [10]. This alcoholate synthesis was first introduced by Nagasaki et al. [11], and later it was called oxyanionic synthesis when further studies of the mechanism were done $[12,13]$. Since then, numerous macroinitiators containing hydroxyl groups have been studied, among them PEO [14], PPO [15], poly(dimethyl siloxane) [16], short aliphatic carbon chains [17] and different combinations of these [14-20]. The oxyanionic method has also been successfully applied to polymerize some other methacrylates, like 2-(trialkylsiloxyethyl) methacrylate [21].

Relatively high concentrations of PEO, PPO, PDM, and their block copolymers can easily be dissolved in water, and their rheological characterization has mainly focused on solution properties [2, 7, 22]. In contrast to the solution properties, their thermomechanical properties have not been studied extensively. Rheological properties of PEO homopolymer have been studied in more detail $[23,24]$ and some crystallization studies of PEO-polystyrene (PS) block co-polymers have been performed with rheometry [25]. Still, the rheological properties of polymers are of interest, and other types of block co-polymers [26, 27], liquid crystalline polymers [28], and star polymers [29] have usually been analysed thoroughly. Rotational rheometry, often together with scattering techniques [30], has been used for studies on microphase separation [31], crystallinity [25], and order to disorder transitions of block co-polymers [32-36].

In this study a set of PEO, PPO and PEO-PPO-PEO macroinitiators was used to synthesize block polymers with PDM segments using oxyanionic synthesis. Materials were characterized with nuclear magnetic resonance (NMR) spectroscopy as well as with size exclusion chromatography (SEC) using a light scattering detector to confirm block formation. Thermal characterization of the resulting polymers was done with differential scanning calorimetry (DSC) and rotational rheometry (RR). Detailed melt rheology of the polymers was studied and the effect of PDM segment on melt rheology is discussed here.

\section{Experimental}

\subsection{Materials}

The molecular structures of the used pre-polymers/macroinitiators are listed in Table 1. PEO and PPO were received from Fluka (Gallen, Switzerland) and PEO-PPO-PEO was from Aldrich (St. Louis, MO, USA). The initiator for PDM homopolymerization was ethylene glycol (EG) from Riedel de Haen (Seelze, Germany). Chemical structures of the different blocks are presented in Figure 1. Other materials and their suppliers were: 2-(dimethylamino) ethyl methacrylate (DMAEMA), potassium hydride $(\mathrm{KH})(30 \mathrm{w} \%$ suspension in mineral oil), and triethyl amine (TEA) from Aldrich (St. Louis, MO, USA); basic alumina, methanol and methylene iodide from Fluka (Gallen, Switzerland); ethanol from Altia (Rajamäki, Finland); diethylether, hexane and acetone from BHD Prolabo (VWR, Karlskoga, Sweden); sodium chloride from Merck (Whitehouse Station, NJ, USA); chloroform $\left(\mathrm{CHCl}_{3}\right)$ from VWR (Karlskoga, Sweden). All chemicals used were of reagent grade.

PEO was purified by precipitation from cold ethanol followed by drying in a vacuum oven overnight [37]. PEO-PPO-PEO and PPO were purified by drying in a vacuum oven overnight. DMAEMA was purified by removing inhibitor by filtration through basic alumina. DMAEMA was stored in a refrigerator before use. All the solvents used in oxyanionic synthesis were dried with molecular sieves for several days. Other chemicals were used without further treatment.

Table 1. Block composition and $\mathrm{dn} / \mathrm{dc}$ values of the prepolymers (in $\mathrm{CHCl}_{3} / \mathrm{TEA}$ (2\%) solution)

\begin{tabular}{|l|r|c|c|c|}
\hline \multirow{2}{*}{\multicolumn{1}{|c|}{ Pre-polymer }} & \multicolumn{2}{|c|}{ Composition [g/mol] } & dn/dc \\
\cline { 2 - 5 } & PEO & PPO & PEO & {$[\mathbf{m l} / \mathbf{g}]$} \\
\hline PEO & 10000 & - & - & 0.060 \\
\hline PEO-PPO-PEO-H (PEO High) & 6000 & 2500 & 6000 & 0.050 \\
\hline PEO-PPO-PEO-L (PEO Low) & 850 & 4100 & 850 & 0.035 \\
\hline PPO & - & 2000 & - & 0.020 \\
\hline
\end{tabular}

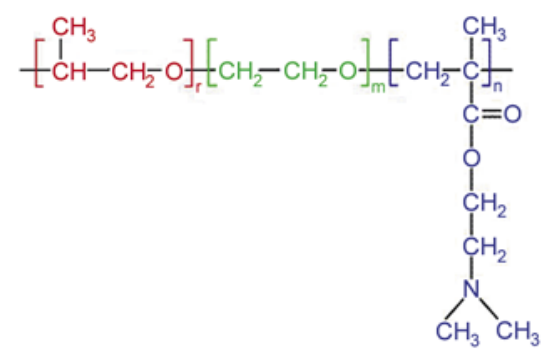

Figure 1. The block structures of PPO (left), PEO (middle), and PDM (right) 


\subsection{Synthesis of block co-polymers}

Amphiphilic water soluble block copolymers were prepared through oxyanionic synthesis. Linear hydroxyl functionalized EG, PEO, PPO or PEOPPO-PEO were activated with potassium hydride $(\mathrm{KH}) . \mathrm{KH}$ reacted with $\mathrm{OH}$-groups and the resulting activated polymer (e.g. K-PEO-K) was used as a macroinitiator. The synthesis was carried out at $25^{\circ} \mathrm{C}$ according to the following example procedure: $10 \mathrm{~g}$ of $\mathrm{PEO}$ and $950 \mathrm{mg}$ of $30 \%$ potassium hydride suspension was added to a dry reaction vessel and argon atmosphere was assured with three vacuum cycles. Under argon atmosphere, $50 \mathrm{ml}$ THF was added to the solution. Homogeneity of the solution was achieved by warming the mixture at $45^{\circ} \mathrm{C}$ for 15 minutes. The vessel was cooled down and the activation reaction was continued for 1.5 hours at ambient temperature (formed $\mathrm{H}_{2}$ removed through a needle). The polymerization was started by adding $15.8 \mathrm{ml}$ of DMAEMA dropwise and carried out for one hour. The reaction was quenched with methanol and precipitated with diethyl ether and hexane. The yield was $13.4 \mathrm{~g}$. The theoretical molecular weight of the PDM block was $7400 \mathrm{~g} / \mathrm{mol}$.

\subsection{Nuclear magnetic resonance spectroscopy (NMR)}

The NMR spectrum was obtained using a $300 \mathrm{MHz}$ Varian Gemini 2000 (Agilent Technologies, Santa Clara, CA, USA) with deuterated chloroform $\left(\mathrm{CDCl}_{3}\right)$ as the solvent. Conversion of the synthesis (decrease of vinylidene groups) was followed with ${ }^{1} \mathrm{H}$ NMR spectroscopy. Polymer structure was identified based on proton peaks of $\mathrm{CH}_{2}(\mathrm{PEO})$ at $3.64 \mathrm{ppm}, \mathrm{N}\left(\mathrm{CH}_{3}\right)_{2}$ (PDM) at $2.27 \mathrm{ppm}$ or $\mathrm{CH}_{3}(\mathrm{PPO})$ at $1.16 \mathrm{ppm}$ [38]. Reacted end groups were detected in quantitative ${ }^{13} \mathrm{C}$ NMR based on absence of a peak at $61.5 \mathrm{ppm}$ in PEO and at 65.5 and 67.1 in PPO.

\subsection{Size exclusion chromatography (SEC)}

Purified polymers were analysed with size exclusion chromatography (SEC) using chloroform with $2 \%$ triethylamine (TEA) as eluent. Elution speed was $1 \mathrm{ml} / \mathrm{min}$ through the following column system: PLgel pre-column and PLgel, $10^{4}, 10^{5}, 10^{3}$ and $10^{2} \AA$ columns supplied by Polymer laboratories (Agilent Technologies, Santa Clara, CA, USA). Relative changes in molecular weight were determined with a Waters RI-detector (refractive index) (Waters,
Milford, MA, USA) against polystyrene standards at $35^{\circ} \mathrm{C}$. A Wyatt Dawn $8^{+}$MALLS (multiangle laser light-scattering) detector (Wyatt Technology, Dernbach, Germany) was used to analyse both molecular weight and size of the polymer. The difference in refractive index between eluent and polymer $(\mathrm{d} n / \mathrm{d} c)$ was estimated assuming $100 \%$ recovery of the known mass concentration through the elution system.

\subsection{Differential scanning calorimetry (DSC)}

The thermal behavior of polymers was measured with a Mettler Toledo DSC $821^{\mathrm{e}}$ (Mettler Toledo, Gerifensee Switzerland) differential scanning calorimeter under a nitrogen atmosphere. The sample size was $7-10 \mathrm{mg}$ for crystalline polymers, and $25 \mathrm{mg}$ for amorphous polymers. Before nonisothermal runs the samples were heated to equalization temperature $\left(150^{\circ} \mathrm{C}\right)$ followed by immediate determination of the crystallization temperature $\left(T_{\mathrm{c}}\right)$ at a cooling rate of $-10^{\circ} \mathrm{C} / \mathrm{min}$ (from 150 to $-100^{\circ} \mathrm{C}$ ). After this cooling step, the melting endotherm $(\Delta H)$, glass transition point $\left(T_{\mathrm{g}}\right)$, and the peak melting temperature $\left(T_{\mathrm{m}}\right)$ were measured by reheating the sample from -100 to $100^{\circ} \mathrm{C}$. The used heating rate was $20^{\circ} \mathrm{C} / \mathrm{min}$ to improve the detectability of $T_{\mathrm{g}}$. In addition to this, the PDM-PEO-PPO-PEO-PDM-L polymer was studied in more detail by determining the influence of the different heating $(5,10,20$ and $\left.40^{\circ} \mathrm{C} / \mathrm{min}\right)$ and cooling rates $(-2,-5,-10$, $-20^{\circ} \mathrm{C} / \mathrm{min}$ ) on the cold crystallization. Also the influence of different equalization temperatures $\left(150,100,80,60,50,40^{\circ} \mathrm{C}\right)$ were studied for the PDM-PEO-PPO-PEO-PDM-L polymer.

\subsection{Rotational rheometry (RR)}

Thermal behaviour of the polymers was measured with an Anton Paar Physica MRC 301 dynamic rotational rheometer (Anton Paar GmbH, Graz, Austria) operated in strain-controlled mode. Temperature was controlled with a Peltier heating element on the lower plate, and cooling was done with a water circulator. $25 \mathrm{~mm}$ plate geometry with gap size of $150 \mu \mathrm{m}$ was used. The dimensional changes in geometry during measurements were taken into account by controlling the gap size with a normal force, and measuring the gap size with a TruGap induction sensor (gap size decreased from 150 to $138 \mu \mathrm{m}$ due to the shrinking of the polymer during the cooling 
ramp). The sample was stabilized at $100^{\circ} \mathrm{C}$ for $30 \mathrm{~min}$ before the measurement. The measured temperature ramp was cooling from 100 to $-30^{\circ} \mathrm{C}$ at $5{ }^{\circ} \mathrm{C} / \mathrm{min}$ followed by immediate heating back to $100^{\circ} \mathrm{C}$ with the same rate. Oscillatory measurement was done at $0.1 \%$ strain within the linear viscoelastic region at $10 \mathrm{~Hz}$ frequency. Moduli and complex viscosity were recorded. Frequency sweeps for time temperature superposition (TTS) calculations were measured from 300 to $0.03 \mathrm{rad} / \mathrm{s}$ with the same gap settings. The TTS measuring temperatures were 40 , $30,20,10,5,0,-5,-10,-15,-20,-25^{\circ} \mathrm{C}(50 \mathrm{~s}$ stabilization between the measurements) and shift factors were calculated by the Rheoplus software according to Williams-Landel-Ferry (WLF) equation [39].

Single temperature frequency sweeps were measured with a stress-controlled rheometer (TA Instruments AR-G2,TA Instruments, New Castle, DE, USA). The measurements were done at $60^{\circ} \mathrm{C}$ or, for polymers without $\mathrm{PPO}$, at $65^{\circ} \mathrm{C}$ with $25 \mathrm{~mm}$ plate geometry using a $250 \mu \mathrm{m}$ gap. The strain used was $0.1 \%$ and the measurement was done with a frequency range of $0.01-100 \mathrm{~Hz}$.

\section{Results and discussion}

\subsection{Synthesis and characterization of block co-polymers}

Oxyanionic polymerization can be carried out in two different ways, namely directly from potassium hydride $(\mathrm{KH})$ or through a $\mathrm{DMSO}^{-} \mathrm{K}^{+}$complex [16] $(\mathrm{DMSO}=$ dimethyl sulfoxide $)$. The $\mathrm{DMSO}^{-} \mathrm{K}^{+}$ method is assumed to be more accurate since the conversion of macroinitiator into $\mathrm{RO}^{-} \mathrm{K}^{+}$can easily be measured with triphenyl methane as an indicator. In this study the initiation from $\mathrm{KH}$ was determined adequate for the polymerization. An excess of $\mathrm{KH}$ was used to eliminate possible termination reactions caused by residual $\mathrm{OH}$ groups [20].
After the synthesis step, all analyses were performed on purified samples as some PDM homopolymer was usually formed in the crude reaction solution. After purification, the formation of block structure was confirmed with ${ }^{13} \mathrm{C} \mathrm{NMR}$, and SEC equipped with MALLS. To obtain reliably molecular weight analysis (using SEC with MALLS detector), the $\mathrm{d} n / \mathrm{d} c$ values of the pre-polymers should be available. There was no literature data available concerning $\mathrm{d} n / \mathrm{d} c$ values for PEO and PPO in $\mathrm{CHCl}_{3} /$ TEA ( $2 \%)$ solution, and therefore the $\mathrm{dn} / \mathrm{dc}$ values were first determined for the whole set of macroinitiators. The values were determined from chromatographic analysis assuming $100 \%$ recovery from the elution system and the scattering data was calculated using a Zimm plot. $\mathrm{dn} / \mathrm{dc}$ values obtained for pre-polymers (Table 1) varied in line with the molecular compositions, so that a $\mathrm{dn} / \mathrm{dc}$ of $0.06 \mathrm{ml} / \mathrm{g}$ was achieved for pure PEO and $0.02 \mathrm{ml} / \mathrm{g}$ for pure PPO. The other macroinitiators gave values between these two depending on block composition. The measured value of $0.02 \mathrm{ml} / \mathrm{g}$ for PPO was rather low but still above the resolution limit of the equipment. $\mathrm{d} n / \mathrm{d} c$ values for modified polymers are listed in Table 2. PDM had the highest $\mathrm{d} n / \mathrm{d} c$ and it was markedly different from the value of 0.02 obtained for macroinitiator PPO. In addition, the experimental $\mathrm{dn} / \mathrm{dc}$ values were consistent with the theoretical values calculated from macroinitiators and PDM homopolymer as averages based on molecular composition.

The results from SEC/MALLS analysis are presented in Table 2 and the formation of PDM block was supported by the increase in molecular weight compared to pre-polymers in Table 1. In addition, the molecular weight distributions of PDM-PEOPDM and PDM-PPO-PDM remained narrow whereas a slightly broader distribution for PDM-PEO-PPOPEO-PDM-H and PDM-PEO-PPO-PEO-PDM-L

Table 2. Molecular weight characterization of the synthesized block polymers using MALLS SEC

\begin{tabular}{|c|c|c|c|c|c|}
\hline \multirow[b]{2}{*}{ Polymer } & \multirow{2}{*}{$\begin{array}{l}\mathrm{dn} / \mathrm{dc}^{\mathrm{a}} \\
{[\mathrm{ml} / \mathrm{g}]}\end{array}$} & \multirow{2}{*}{$\begin{array}{c}\mathrm{dn} / \mathrm{dc}^{\mathrm{b}} \\
{[\mathrm{ml} / \mathrm{g}]}\end{array}$} & \multicolumn{3}{|c|}{ Molecular weight [g/mol] } \\
\hline & & & $M_{n}$ & $\mathbf{M}_{\mathbf{w}}$ & PD \\
\hline PDM & 0.070 & 0.07 & 14600 & 19900 & 1.37 \\
\hline PDM-PEO-PDM & 0.064 & 0.07 & 26500 & 28300 & 1.07 \\
\hline PDM-PEO-PPO-PEO-PDM-H & 0.057 & 0.06 & 32300 & 48400 & 1.50 \\
\hline PDM-PEO-PPO-PEO-PDM-L & 0.055 & 0.06 & 11000 & 17000 & 1.55 \\
\hline PDM-PPO-PDM & 0.060 & 0.06 & 13900 & 14300 & 1.03 \\
\hline
\end{tabular}

${ }^{\mathrm{a}}$ theoretical values

$b_{\text {values from SEC }}$ 
was observed. Finally, successful initiation of the end groups was detected in ${ }^{13} \mathrm{C}$ NMR as an absence of peaks at $61.5 \mathrm{ppm}\left(\mathrm{PEO}, \mathrm{CH}_{2}-\mathrm{OH}\right)$ or at 65.5 and $67.1 \mathrm{ppm}\left(\mathrm{PPO}, \underline{\mathrm{C}}\left(\mathrm{CH}_{3}\right) \mathrm{H}-\mathrm{OH}\right.$ and $\underline{\mathrm{CH}}_{2}-\mathrm{OH}$ respectively).

\subsection{Thermal transition temperatures with DSC}

Thermal transition temperatures analysed with the DSC are presented in Table 3 and in Figures 2-4. PPO is completely amorphous, with a glass transition point at $-67^{\circ} \mathrm{C}$ (Figure $2 \mathrm{~A}$ ), whereas pure $\mathrm{PEO}$ is a highly crystalline polymer which melts at $66^{\circ} \mathrm{C}$ (Figure $3 \mathrm{C}$ ). The melting temperatures of the crystalline $\mathrm{PEO}$ phases in their block copolymers, PEOPPO-PEO-H and PEO-PPO-PEO-L, are lower (Figure $3 \mathrm{D}$ and $4 \mathrm{~A}, 61$ and $39^{\circ} \mathrm{C}$, respectively) but this is mostly due to the shorter PEO block sizes (Table 1, 6000 and $850 \mathrm{~g} / \mathrm{mol}$, respectively: e.g. PEO $1000 \mathrm{~g} / \mathrm{mol}$ from Fluka, $T_{\mathrm{m}} 37-40^{\circ} \mathrm{C}$ ). In addition, the $T_{\mathrm{g}}$ of the PPO phase is only slightly influenced by the amount of PEO. These results indicate that PEO and PPO were phase separated at solid state.

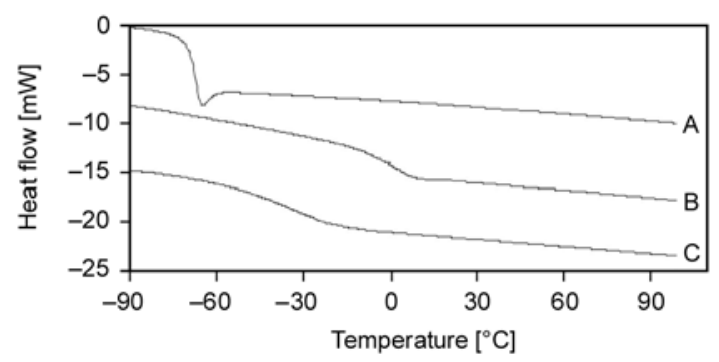

Figure 2. Glass transition points observed in DSC heating sequences for the amorphous polymers, A) PPO pre-polymer, B) PDM reference, and C) PDMPPO-PDM
The reference PDM homopolymer was found to be totally amorphous with $T_{\mathrm{g}}$ at $1{ }^{\circ} \mathrm{C}$ which is in consistent with the literature [40]. However, this thermal transition was not as clear as that of the amorphous PPO (Figure 2 B vs. A). This phenomenon was observed also in their amorphous block copolymer, PDM-PPO-PDM, and only a modest $T_{\mathrm{g}}$ at about $-20^{\circ} \mathrm{C}$ (Figure $2 \mathrm{C}$ ) could be observed. In addition, no separate $T_{\mathrm{g}}$ from PPO was observed which indicates that the PDM and PPO phases were most likely mixed in the solid PDM-PPO-PDM block copolymer.

Modification of the highly crystalline PEO and PEO-PPO-PEO-H polymers with PDM-blocks affected the transition temperatures slightly (Table 3 , Figure 3). $T_{\mathrm{m}}$ and $T_{\mathrm{c}}$ of the crystalline PEO phase were reduced $5-8^{\circ} \mathrm{C}$ but the crystallinity $(\Delta H)$ was remained mostly at the same level in modified polymers. It was clear that the PDM-blocks did not prevent the crystallization of the PEO-segments, which usually indicates that the phases are separated in the solid polymer [29]. Additionally, no glass transition point either due to the amorphous PPO or PDM phase was observed. This is consistent with the results above, which indicated that the $T_{\mathrm{g}}$ is less distinct in polymers containing both PDM and PPO segments.

The most interesting behaviour was found with PDM-PEO-PPO-PEO-PDM-L (Figure 4 B). According to DSC, initially the polymer behaved like a completely amorphous polymer as no crystallization exotherm was observed in the cooling sequence from 150 to $-100^{\circ} \mathrm{C}$. However, in the heating sequence, a clear crystallization peak $\left(T_{\mathrm{c}}\right)$ was found followed immediately by a melting peak. In gen-

Table 3. Thermal transition temperatures and enthalpies $(\Delta H)$ of the studied block-copolymers. Values in parenthesis are thermal transitions observed with rheometer.

\begin{tabular}{|c|c|c|c|c|}
\hline \multirow[b]{2}{*}{ Pre-polymer } & \multicolumn{4}{|c|}{ DSC (Rheometer) } \\
\hline & $\begin{array}{c}\mathbf{T}_{\mathbf{g}} \\
{\left[{ }^{\circ} \mathbf{C}\right]}\end{array}$ & $\begin{array}{c}\mathbf{T}_{\mathbf{c}} \\
{\left[{ }^{\circ} \mathbf{C}\right]}\end{array}$ & $\begin{array}{c}\mathbf{T}_{\mathbf{m}} \\
{\left[{ }^{\circ} \mathbf{C}\right]}\end{array}$ & $\begin{array}{c}\Delta \mathbf{H}_{\text {PEO-phase }} \\
{[\mathbf{J} / \mathrm{g}]}\end{array}$ \\
\hline PEO & - & 41 & $66(62)$ & 180 \\
\hline PEO-PPO-PEO-H & -61 & 39 & $61(58)$ & 150 \\
\hline PEO-PPO-PEO-L & -65 & 17 & $39(38)$ & 170 \\
\hline $\mathrm{PPO}$ & -67 & - & - & \\
\hline \multicolumn{5}{|l|}{ Reference } \\
\hline PDM & $1(5)$ & - & - & \\
\hline \multicolumn{5}{|l|}{ Polymer } \\
\hline PDM-PEO-PDM & - & 35 & $58(53)$ & 220 \\
\hline PDM-PEO-PPO-PEO-PDM-H & - & 34 & $53(49)$ & 180 \\
\hline PDM-PEO-PPO-PEO-PDM-L & -60 & $-12(-15)$ & $14(10)$ & 48 \\
\hline PDM-PPO-PDM & $-20(-10)$ & - & - & \\
\hline
\end{tabular}



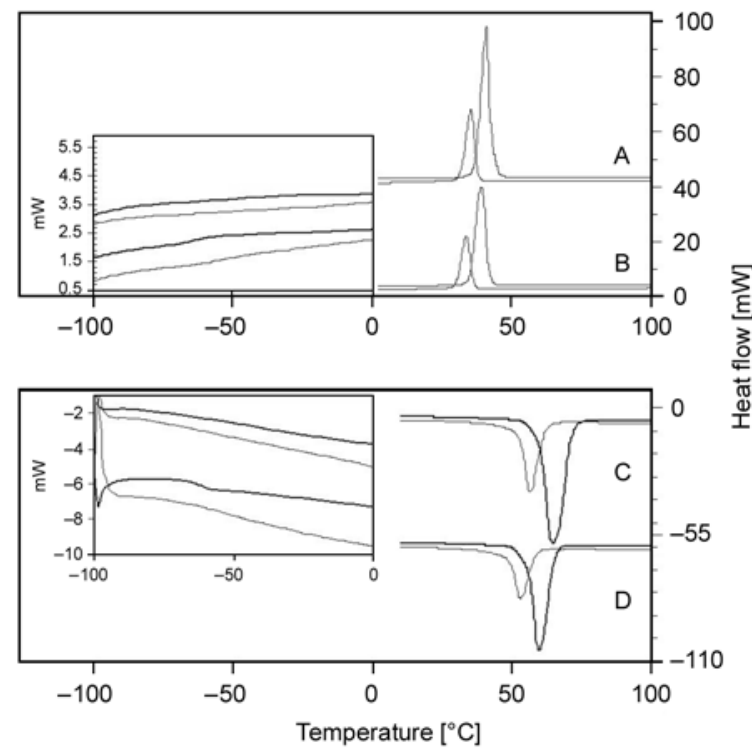

Figure 3. DSC cooling curves of A) PEO, PDM-PEO-PDM B) PEO-PPO-PEO-H, PDM-PEO-PPO-PEOPDM-H. DSC heating curves of C) PEO, PDMPEO-PDM D) PEO-PPO-PEO-H, PDM-PEOPPO-PEO-PDM-H. The thicker curves are for PEO and PEO-PPO-PEO-H.

eral, this kind of behaviour is known as cold crystallization [41] and is typical for polymers [42] and blends [43] that contain both soft and hard segments, as is the case for the PDM-PEO-PPO-PEOPDM-L structure. Cold crystallization is also often dependent on heating/cooling rate [44], which, however, was not clearly the case in this polymer. The influence of cooling rate was minor (Figure 4 $\mathrm{B}, \mathrm{C}, \mathrm{D})$ whereas the $\Delta H$ were slightly affected by varying the heating rate (Figure $4 \mathrm{E} \Delta H=10 \mathrm{~J} / \mathrm{g}$; $4 \mathrm{~F} \Delta H=6 \mathrm{~J} / \mathrm{g}$ ). Finally, when the equalization temperature was reduced from 150 to below $50^{\circ} \mathrm{C}$, the cold crystallization was absent and only melting endotherm (Figure $4 \mathrm{G}, T_{\mathrm{m}}$ at $\sim 10^{\circ} \mathrm{C}$ ) was observed during heating. Still, this melting endotherm differed largely when compared with the one obtained for unmodified PEO-PPO-PEO-L $\left(T_{\mathrm{m}} \sim 10^{\circ} \mathrm{C}\right.$ vs. $39^{\circ} \mathrm{C} ; \Delta H_{\text {PEO-phase }} 48 \mathrm{~J} / \mathrm{g}$ vs $170 \mathrm{~J} / \mathrm{g}$ ).

One explanation to the thermal behaviour of PDMPEO-PPO-PEO-PDM-L can be the strong phase mixing at melt state (equalization temperature $50^{\circ} \mathrm{C}$ or higher), which hindered the homogeneous and/or heterogeneous nuclei formation during the cooling sequence. As well known the absence of any nuclei will effectively prevent the crystallization of the polymer phase. However, when the PDM-PEOPPO-PEO-PDM-L was equalized only at $40^{\circ} \mathrm{C}$, the

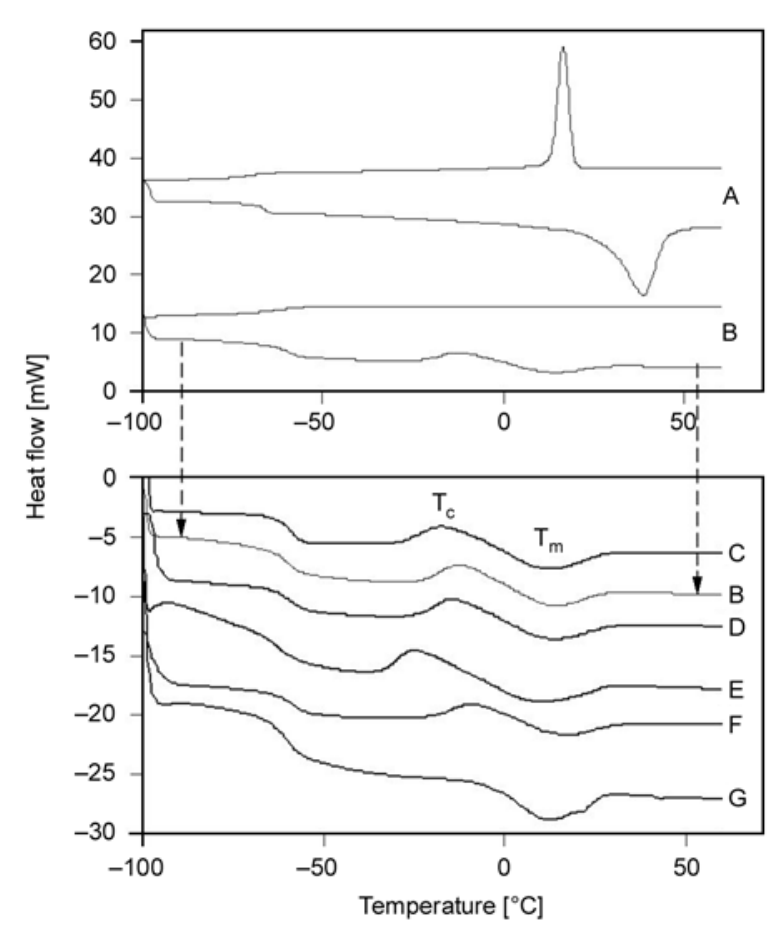

Figure 4. DSC heating and cooling curves of A) PEO-PPOPEO-L pre-polymer, B) PDM-PEO-PPO-PEOPDM-L. Heating curves of PDM-PEO-PPO-PEOPDM-L with varying parameters, C) cooling $-2^{\circ} \mathrm{C} / \mathrm{min}$, heating $20^{\circ} \mathrm{C} / \mathrm{min}$ B) cooling $-10^{\circ} \mathrm{C} / \mathrm{min}$, heating $20^{\circ} \mathrm{C} / \mathrm{min}$, D) cooling $-20^{\circ} \mathrm{C} / \mathrm{min}$, heating $20^{\circ} \mathrm{C} / \mathrm{min}$, E) cooling $-10^{\circ} \mathrm{C}$, heating $5^{\circ} \mathrm{C} / \mathrm{min}$, and $\mathrm{F}$ ) cooling $-10^{\circ} \mathrm{C}$, heating $40^{\circ} \mathrm{C} / \mathrm{min}$, G) cooling $-10^{\circ} \mathrm{C} / \mathrm{min}$, heating $20^{\circ} \mathrm{C} / \mathrm{min}$ after the thermal history was equalized at $40^{\circ} \mathrm{C}$.

polymer melt contained probably some unmelted domains which then acted as nucleation centres supporting the crystallization during the cooling sequence. This hypothesis can be associated with the results of Hillmyer and Bates [45] where the melted polyalkane-block-PEO copolymer was found to maintain small crystalline PEO fractions.

\subsection{Thermal transition temperatures measured by rheometry}

Rotational rheometry was also used to analyse thermal transition behaviour. However, crystallization kinetics can cause problems when measuring temperature transitions with rheometry. Strain-induced crystallization can be problem when a stress-controlled instrument is used [46]. That can lead to high strains and differences in the rate of crystallization measured by rheometry versus DSC. In this study the strain-controlled measurement was performed at very low strain, hence mitigating the risk 

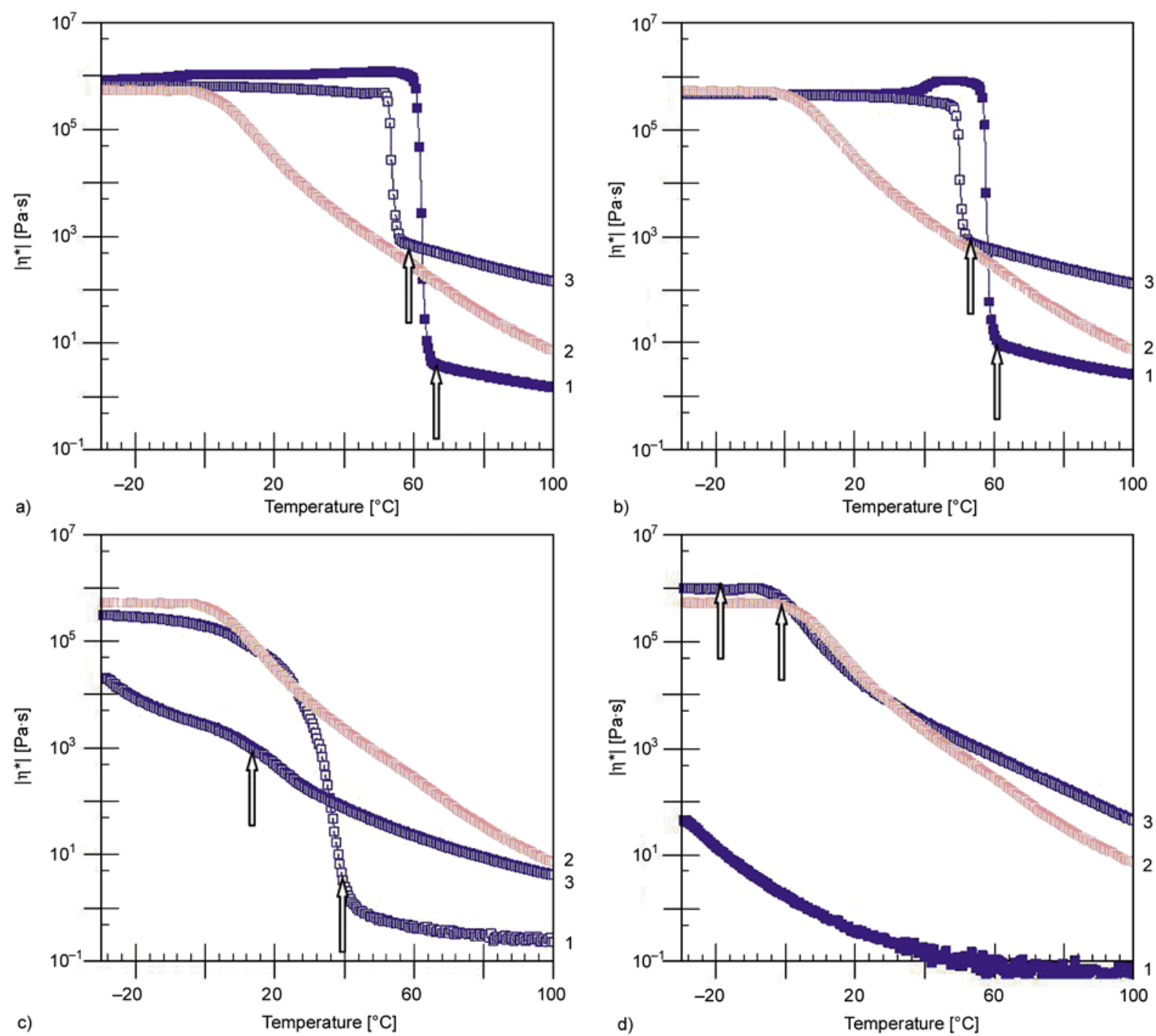

Figure 5. Effect of PDM on thermal transition behaviour observed as complex viscosity of a) $1=$ PEO, $2=$ PDM, $3=$ PDMPEO-PDM; b) 1 = PEO-PPO-PEO-H, 2 = PDM, 3 = PDM-PEO-PPO-PEO-PDM-H; c) 1 = PEO-PPO-PEO-L, $2=$ PDM, 3 = PDM-PEO-PPO-PEO-PDM-L; d) 1 = PPO, 2 = PDM, 3 = PDM-PPO-PDM. Thermal transition points determined with DSC are marked with arrows.

of strain-induced crystallization. In addition, the ability to controlled strain instead of stress produced repeatable results, and sensitivity was additionally increased by using $10 \mathrm{~Hz}$ frequency.

The problem with temperature scans can be that hard polymers may be outside equipment's maximum torque range. In our case, however, the polymers were soft enough so that the melting temperatures as well the glass transition points were clearly obtained as decrease in complex viscosity (Figures $5 \mathrm{a}-\mathrm{d}$ ). The obtained transition temperatures were consistent with the DSC results even though a slight systematic deviation of few degrees was observed between the results (Table 3 ). In addition, the $T_{\mathrm{g}}$ 's of PDM and PDM-PPO-PDM were more distinct when compared with the DSC analysis. Still, either the rheometer was not able to detect separate $T_{\mathrm{g}}$ originating from PDM in any of the polymers containing PEO. The absence of $T_{\mathrm{g}}$ is consistent with the very high viscosity values for PDM-PEO-PDM and PDM-PEO-PPO-PEO-PDM$\mathrm{H}$ obtained below the melting point. At the temperature where the glassy stage of PDM should be softened, PEO is still strongly crystalline with high viscosity, thus binding the PDM and preventing softening. Finally, in addition to the basic thermal transitions, a slight but clear increase in viscosity of solid PEO-PPO-PEO-H was found around $40^{\circ} \mathrm{C}$ 

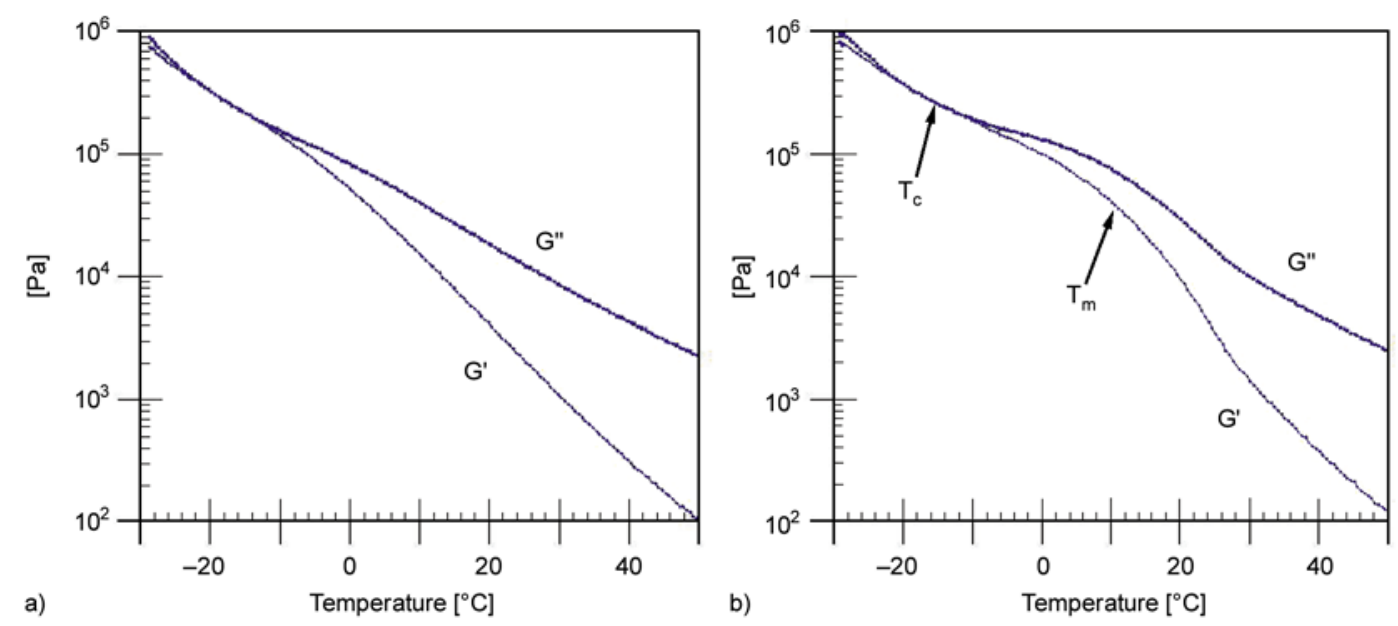

Figure 6. Thermal transition behaviour of storage $\left(G^{\prime}\right)$ and loss $\left(G^{\prime \prime}\right)$ modulus in cooling (a) and heating ramp (b) of PDMPEO-PPO-PEO-PDM-L

(Figure 5 b 1). This kind of increase in viscosity may indicate microphase separation or order disorder transition point [32].

The cold crystallization behaviour PDM-PEOPPO-PEO-PDM-L observed in DSC was studied in detail by comparing storage module $\left(G^{\prime}\right)$ and loss module $\left(G^{\prime \prime}\right)$ in cooling and heating cycles (Figure 6). Just like in DSC, the cold crystallization behaviour was seen only in heating cycle whereas cooling ramp did not indicate any phase transitions occurring. $T_{\mathrm{c}}$ was determined as tangential point of $G^{\prime}$ and $G^{\prime \prime}$ whereas $T_{\mathrm{m}}$ could be seen as clear decrease in modulus. It was also remarkable that regardless of the thermal transition points, $G^{\prime \prime}$ was practically higher than $G^{\prime}$ over the whole range of temperatures. That indicates that this polymer behaves as a high viscosity fluid. This kind of rheological behaviour is normally seen in polymer melts containing some crystalline parts [34].

\subsection{Melt rheology}

Modification of the pre-polymers with PDM-blocks clearly increased melt viscosity of one to three orders of magnitude as can be observed in Figure 5. Still, the weak temperature/viscosity dependence (at the melt state) of the pre-polymers was maintained in the modified polymers. It must also be noted that the melt viscosity of PDM-containing
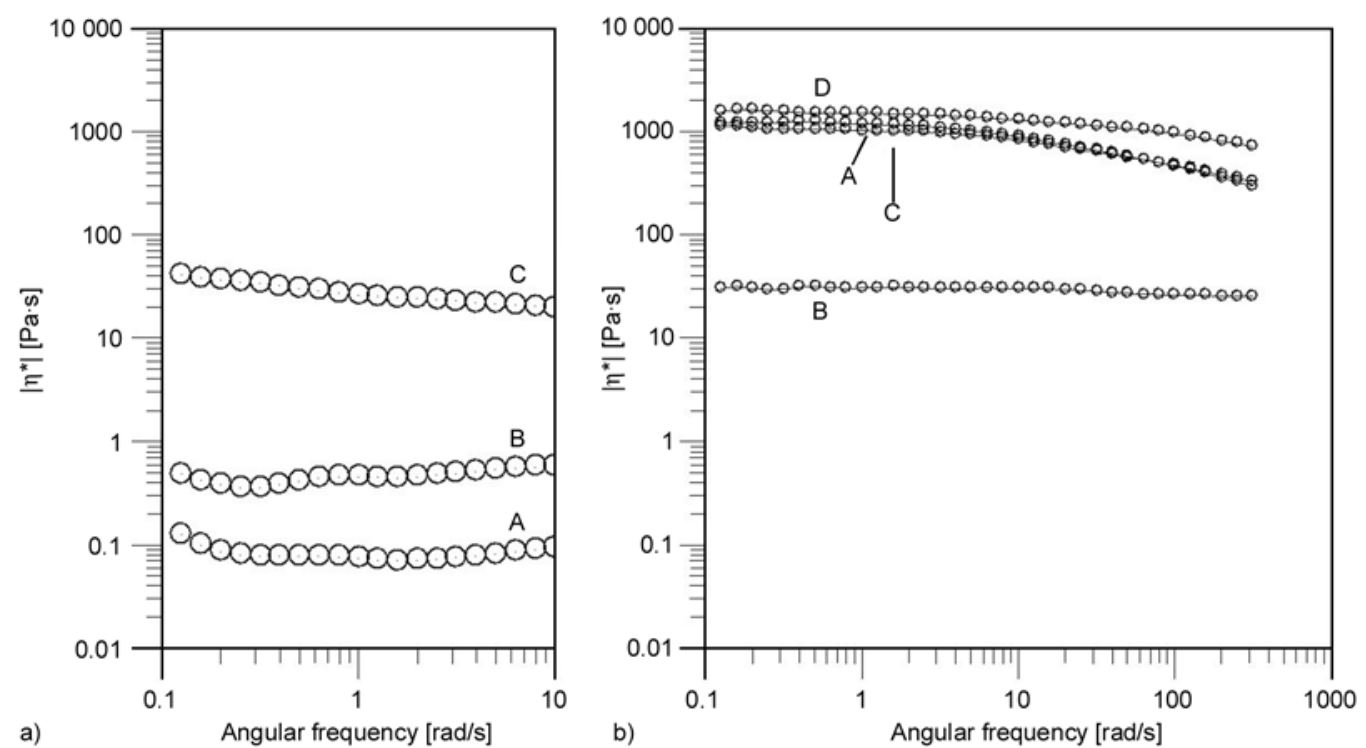

Figure 7. Complex viscosity of pre-polymers (a): $\mathrm{A}=\mathrm{PPO}, \mathrm{B}=\mathrm{PEO}-\mathrm{PPO}-\mathrm{PEO}-\mathrm{L}, \mathrm{C}=\mathrm{PEO}-\mathrm{PPO}-\mathrm{PEO}-\mathrm{H}$ and modified block co-polymers (b): A = PDM-PPO-PDM, B = PDM-PEO-PPO-PEO-L, C = PDM-PEO-PPO-PEO-H, $\mathrm{D}=\mathrm{PDM}$ 


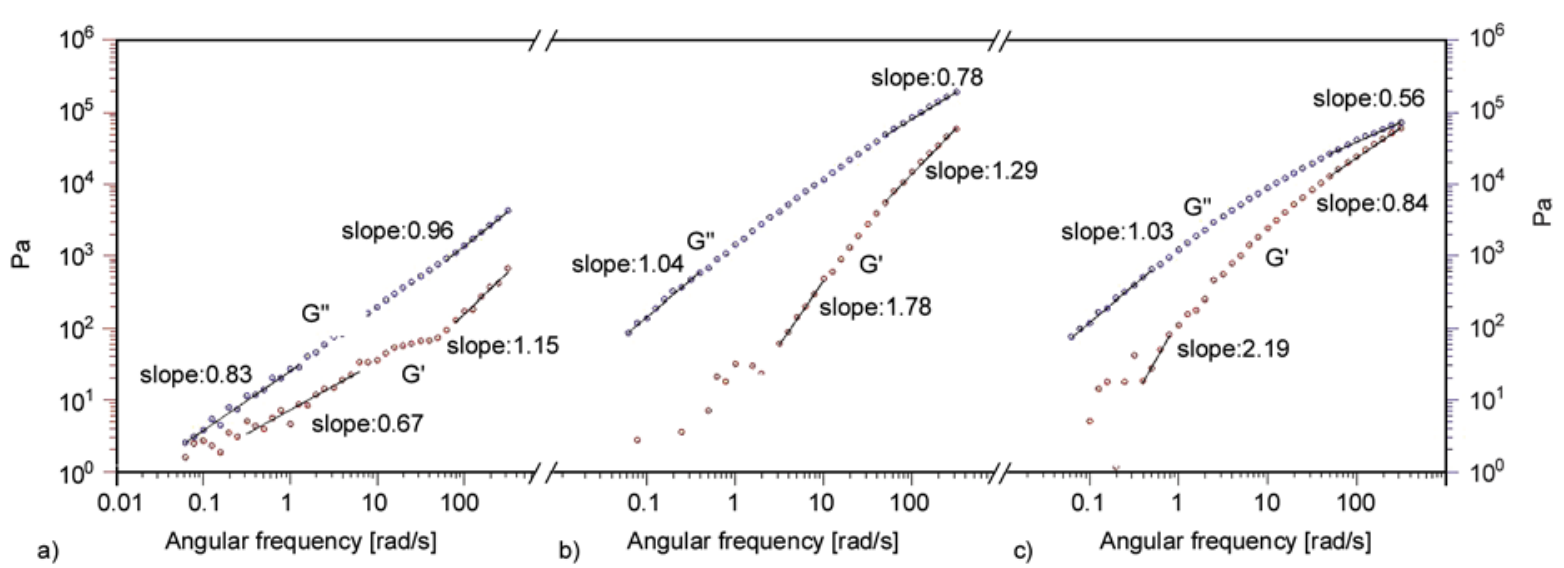

Figure 8. Frequency sweeps of a) PEO-PPO-PEO-H, b) PDM and c) PDM-PEO-PPO-PEO-PDM-H measured at $60^{\circ} \mathrm{C}$

polymer was higher than the average of PDM and the corresponding pre-polymer in all polymers except PDM-PEO-PPO-PEO-PDM-L.

In frequency sweeps the complex viscosity of the pre-polymers showed only a weak frequency dependency indicating strong Newtonian behaviour (Figure 7). This is consistent with what is reported in the literature [24] and indicates that PEO and PPO do not form entanglements at the melt stage. However, these polymers had also measurable storage modulus (Figure $8 \mathrm{a}$ and $\mathrm{b}$ ), which is slightly contradictory with the assumption of Newtonian fluid. Modification of the pre-polymers (excluding PEOPPO-PEO-L) with PDM was seen as the appearance of a shear thinning character (Figure 7) and with an obvious elastic modulus (Figure 8c). The module dependence of angular frequency $(\omega)$ was calculated and correlations $G^{\prime \prime} \sim \omega^{1}$ and $G^{\prime} \sim \omega^{2}$ were observed at low shear rates (slopes in Figure 8c). This terminal slope was similar to what is generally seen for entangled polymer melts [34]. Still, no cross-over point for the moduli was observed, but this was due to the limited experimental window available.

\subsection{Time temperature superposition (TTS) frequency sweeps for PDM-PEO-PPO-PEO-PDM-L}

As mentioned above, the PDM-PEO-PPO-PEOPDM-L seemed to act as a Newtonian fluid (at $60^{\circ} \mathrm{C}$ ) even though the other PDM-modified prepolymers exhibited clear shear thinning behaviour. Therefore the TTS was calculated for PDM-PEOPPO-PEO-PDM-L in order to find changes in material characteristics over a wider range of frequen- cies. Unfortunately, the TTS was not suitable for polymers that exhibit strong phase transition at the used temperature range [23], and therefore the direct comparison e.g. with unmodified PEO-PPOPEO-L was not performed.

The TTS measurements for PDM-PEO-PPO-PEOPDM-L were performed from 40 to $-25^{\circ} \mathrm{C}$ to avoid the cold crystallization. The TTS calculations were referenced at $35^{\circ} \mathrm{C}$ (Figure 9) and a clear shear thinning behaviour for PDM-PEO-PPO-PEO-PDM-L was observed. The complex viscosity decreased in parallel of increase in shear force, and a low frequency behaviour $\left(G^{\prime \prime} \sim \omega^{1}\right.$ and $\left.G^{\prime} \sim \omega^{2}\right)$ typical for entangled polymer melts was present. Also a clear cross-over point for $G^{\prime}$ and $G^{\prime \prime}$ was found in frequency ramps at around $100000 \mathrm{rad} / \mathrm{s}$. The earlier Newtonian behaviour of PDM-PEO-PPO-PEO-

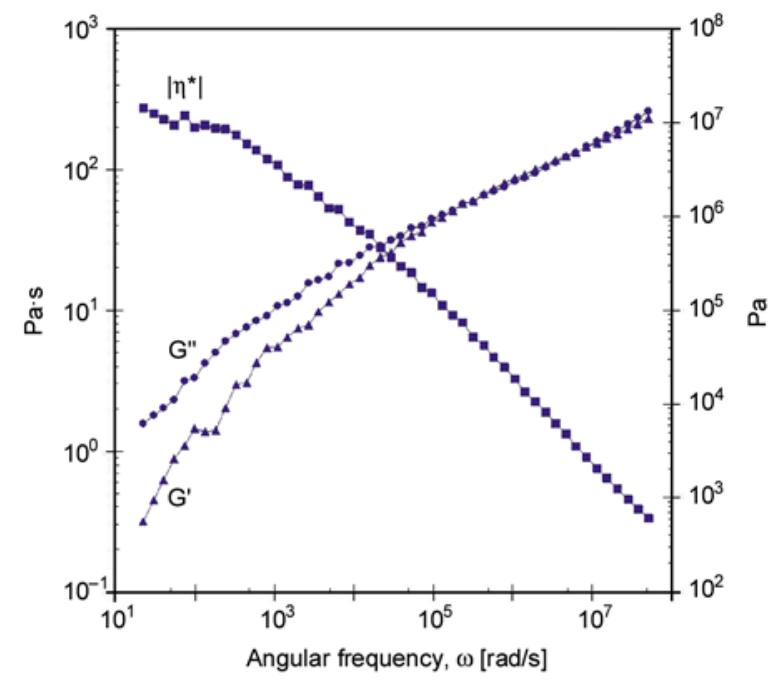

Figure 9. Frequency dependence expressed as complex viscosity $\left(\eta^{*}\right)$, storage modulus $\left(G^{\prime}\right)$ and loss modulus $\left(G^{\prime \prime}\right)$ of PDM-PEO-PPO-PEO-PDM-L. Horizontal shift factor $a_{\mathrm{T}}=0.2468$ and vertical shift factor $b_{\mathrm{T}}=2.039$. Reference temperature is $35^{\circ} \mathrm{C}$. 


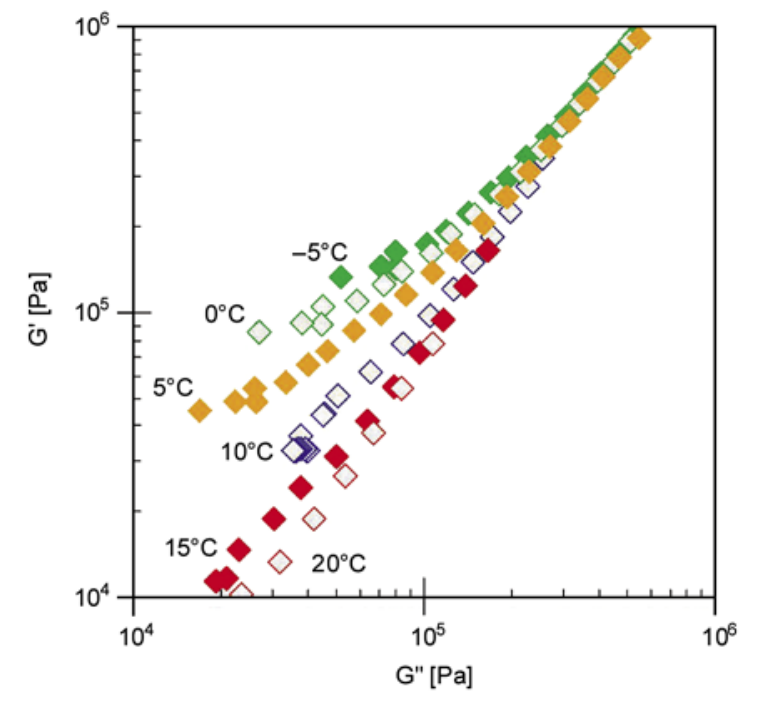

Figure 10. $G^{\prime \prime}$ of PDM-PEO-PPO-PEO-PDM-L plotted against $G^{\prime}$ in frequency sweeps at different temperatures

PDM-L (Figure 7) originated most likely due to the high measuring temperature, where the complex viscosity was at the Newtonian plateau over the whole range of frequencies.

The abovementioned TTS measurements were performed also by starting at the lowest temperature when the cold crystallization of PDM-PEO-PPOPEO-PDM-L was present. Because of occurring phase transition the master curve could not be done, and instead Cole-Cole correlation [33] was plotted (Figure 10, temperature range from -5 to $20^{\circ} \mathrm{C}$ ). At high frequency area hardly any deviations in the ratio of $G^{\prime}$ and $G^{\prime \prime}$ could be observed between the temperatures, but at low frequencies the impact of $G^{\prime}$ gets stronger at lower temperatures. This is a clear indication of occurring phase transition [29], but the detailed microstructure still remains unclear.

\section{Conclusions}

Block polymers were successfully synthesized by using a potassium functionalized pre-polymer as initiator. The formation of block structure was confirmed with SEC and NMR. Thermal transitions were determined by calorimetric and rheological means, and the results were consistent with each other. The thermal behaviour, with obvious glass transitions and melting endotherms, indicated that poly(ethylene oxide) (PEO) and poly(propylene oxide) (PPO) segments are mostly phase separated in these copolymers at solid state. Modification with poly[2-(dimethylamino)ethyl methacrylate] (PDM) did affect the thermal transition temperatures of the PEO-rich pre-polymer only slightly, whereas more significant changes were observed in PPO-rich polymers. Thermal behaviour indicates that PDM is phase separated from PEO but forms a mixed phase with PPO. Cold crystallization behaviour for PPO-rich PDM-PEO-PPO-PEO-PDM-L could be observed in DSC measurements as well as in temperature scan in rheometer.

Melt rheology of the block copolymers confirmed that PEO and PPO block co-polymers behaved almost as Newtonian liquids over the whole range of frequencies. Rheological characterization of the block co-polymers also confirmed that melt viscosity of the pre-polymers was increased by modifying them with PDM block. Additionally, PDM segments seemed to increase frequency dependence of the polymers slightly and to modify polymers from Newtonian into shear thinning fluids. Overall, the frequency sweeps confirmed the behaviour seen in temperature scans. No cross-over frequency was found for polymers without PDM, but the elastic component originating from PDM can give advantage in melt processing of these polymers.

\section{References}

[1] Lodge T. P.: Block copolymers: Past successes and future challenges. Macromolecular Chemistry and Physics, 204, 265-273 (2003).

DOI: $10.1002 / \mathrm{macp} .200290073$

[2] Mortensen K., Batsberg W., Hvidt S.: Effects of PEO-PPO diblock impurities on the cubic structure of aqueous PEO-PPO-PEO pluronics micelles: fcc and bcc ordered structures in F127. Macromolecules, 41, 1720-1727 (2008).

DOI: $10.1021 / \mathrm{ma} 702269 \mathrm{c}$

[3] Ibrahim K., Salminen A., Holappa S., Kataja K., Lampinen H., Löfgren B., Laine J., Seppälä J.: Preparation and characterization of polystyrene-poly(ethylene oxide) amphiphilic block copolymers via atom transfer radical polymerization: Potential application as paper coating materials. Journal of Applied Polymer Science, 102, 4304- 4313 (2006).

DOI: $10.1002 / a p p .24886$

[4] Nurmi L., Holappa S., Nykänen A., Laine J., Ruokolainen J., Seppälä J.: Ultra-thin films of cationic amphiphilic poly(2-(dimethylamino)ethyl methacrylate) based block copolymers as surface wettability modifiers. Polymer, 50, 5250-5261 (2009).

DOI: $10.1016 /$ j.polymer.2009.08.043 
[5] Vesterinen A-H., Rich J., Myllytie P., Laine J., Seppälä J.: Poly(ethylene oxide)-block-poly[2-(dimethylamino) ethyl methacrylate] as strengthening agent in paper: Dynamic mechanical characterization. Macromolecular Materials and Engineering, 295, 269-275 (2010). DOI: $10.1002 /$ mame.200900317

[6] Vesterinen A-H., Rich J., Seppälä J.: Synthesis and solution rheology of poly[(stearyl methacrylate)-stat([2-(methacryloyloxy)ethyl] trimethyl ammonium iodide)]. Journal of Colloid and Interface Science, 351, 478-484 (2010).

DOI: $\underline{10.1016 / j . j c i s .2010 .07 .073}$

[7] Peng Z., Li G., Liu X., Tong Z.: Synthesis, pH- and temperature-induced micellization and gelation of doubly hydrophilic triblock copolymer of $\operatorname{poly}(N, N$ dimethylamino-2-ethylmethacrylate)- $b$-poly(ethylene glycol)- $b$-poly( $N, N$-dimethylamino-2-ethylmethacrylate) in aqueous solutions. Journal of Polymer Science Part A: Polymer Chemistry, 46, 5869-5878 (2008). DOI: $10.1002 /$ pola.22904

[8] Baines F. L., Billingham N. C., Armes S. P.: Synthesis and solution properties of water-soluble hydrophilichydrophobic block copolymers. Macromolecules, 29, 3416-3420 (1996).

DOI: $10.1021 / \mathrm{ma} 951699+$

[9] Zeng F., Shen Y., Zhu S., Pelton R.: Synthesis and characterization of comb-branched polyelectrolytes. 1 . Preparation of cationic macromonomer of 2-(dimethylamino)ethyl methacrylate by atom transfer radical polymerization. Macromolecules, 33, 1628-1635 (2000).

DOI: $10.1021 / \mathrm{ma} 991654 \mathrm{z}$

[10] de Paz Báñez M. V., Robinson K. L., Bütün V., Armes S. P.: Use of oxyanion-initiated polymerization for the synthesis of amine methacrylate-based homopolymers and block copolymers. Polymer, 42, 29-37 (2000). DOI: 10.1016/S0032-3861(00)00329-3

[11] Nagasaki Y., Sato Y., Kato M.: A novel synthesis of semitelechelic functional poly(methacrylate)s through an alcoholate initiated polymerization. Synthesis of poly[2-( $N, N$-diethylaminoethyl) methacrylate] macromonomer. Macromolecular Rapid Communications, 18, 827-835 (1997).

DOI: $10.1002 /$ marc.1997.030180911

[12] Vamvakaki M., Billingham N. C., Armes S. P.: Synthesis of controlled structure water-soluble diblock copolymers via oxyanionic polymerization. Macromolecules, 32, 2088-2090 (1999).

DOI: $10.1021 / \mathrm{ma} 9817790$

[13] Lascelles S. F., Malet F., Mayada R., Billingham N. C., Armes S. P.: Latex syntheses using novel tertiary amine methacrylate-based macromonomers prepared by oxyanionic polymerization. Macromolecules, 32, 2462-2471 (1999).

DOI: $10.1021 / \mathrm{ma981967e}$
[14] Tong D., Yao J., Li H., Han S.: Synthesis and characterization of thermo- and $\mathrm{pH}$-sensitive block copolymers bearing a biotin group at the poly(ethylene oxide) chain end. Journal of Applied Polymer Science, 102, 3552-3558 (2006).

DOI: 10.1002/app.24812

[15] Ni P-H., Pan Q-S., Zha L-S., Wang C-C., Elaïssari A., Fu S-K.: Syntheses and characterizations of poly[2(dimethylamino)ethyl methacrylate]-poly(propylene oxide)-poly[2-(dimethylamino)ethyl methacrylate] ABA triblock copolymers. Journal of Polymer Science Part A: Polymer Chemistry, 40, 624-631 (2002). DOI: $10.1002 /$ pola.10144

[16] de Paz Báñez M. V., Robinson K. L., Armes S. P.: Synthesis and solution properties of dimethylsiloxane-2(dimethylamino)ethyl methacrylate block copolymers. Macromolecules, 33, 451-456 (2000). DOI: $10.1021 / \mathrm{ma991665 \textrm {s }}$

[17] de Paz Báñez M. V., Robinson K. L., Vamvakaki M., Lascelles S. F., Armes S. P.: Synthesis of novel cationic polymeric surfactants. Polymer, 41, 8501-8511 (2000). DOI: $10.1016 / \mathrm{S} 0032-3861(00) 00217-2$

[18] Jin L., Deng Y., Hu J., Wang C.: Preparation and characterization of core-shell polymer particles with protonizable shells prepared by oxyanionic polymerization. Journal of Polymer Science Part A: Polymer Chemistry, 42, 6081-6088 (2004).

DOI: $10.1002 /$ pola.20453

[19] Li Y., Armes S. P.: Synthesis of branched water-soluble vinyl polymers via oxyanionic polymerization. Macromolecules, 38, 5002-5009 (2005).

DOI: $10.1021 / \mathrm{ma} 050333 \mathrm{~m}$

[20] Anderson B. C., Cox S. M., Bloom P. D., Sheares V. V., Mallapragada S. K.: Synthesis and characterization of diblock and gel-forming pentablock copolymers of tertiary amine methacrylates, poly(ethylene glycol), and poly(propylene glycol). Macromolecules, 36, 1670-1676 (2003).

DOI: $10.1021 / \mathrm{ma} 0211481$

[21] Iijima M., Nagasaki Y., Kato M., Kataoka K.: A potassium alcoholate-initiated polymerization of 2-(trialkylsiloxyethyl) methacrylate. Polymer, 38, 11971202 (1997).

DOI: $10.1016 / \mathrm{S} 0032-3861(96) 00623-4$

[22] Ebagninin K. W., Benchabane A., Bekkour K.: Rheological characterization of poly(ethylene oxide) solutions of different molecular weights. Journal of Colloid and Interface Science, 336, 360-367 (2009). DOI: $10.1016 /$ j.jcis.2009.03.014

[23] Niedzwiedz K., Wischnewski A., Pyckhout-Hintzen W., Allgaier J., Richter D., Faraone A.: Chain dynamics and viscoelastic properties of poly(ethylene oxide). Macromolecules, 41, 4866-4872 (2008).

DOI: $10.1021 / \mathrm{ma} 800446 \mathrm{n}$ 
[24] Coppola S., Grizzuti N., Floudas G., Vlassopoulos D.: Viscoelasticity and crystallization of poly(ethylene oxide) star polymers of varying arm number and size. Journal of Rheology, 51, 1007-1025 (2007).

DOI: $10.1122 / 1.2751076$

[25] Floudas G., Tsitsilianis C.: Crystallization kinetics of poly(ethylene oxide) in poly(ethylene oxide)-polystyrene-poly(ethylene oxide) triblock copolymers. Macromolecules, 30, 4381-4390 (1997).

DOI: $10.1021 / \mathrm{ma9616118}$

[26] Yurekli K., Krishnamoorti R.: Dynamics of block copolymer micelles. Macromolecules, 35, 4075-4083 (2002).

DOI: $10.1021 / \mathrm{ma} 012024 \mathrm{~b}$

[27] Sebastian J. M., Lai C., Graessley W. W., Register R. A., Marchand G. R.: Steady-shear rheology of block copolymer melts: Zero-shear viscosity and shear disordering in body-centered-cubic systems. Macromolecules, 35, 2700-2706 (2002).

DOI: $10.1021 / \mathrm{ma} 011522 \mathrm{~h}$

[28] Wewerka A., Floudas G., Pakula T., Stelzer F.: Sidechain liquid-crystalline homopolymers and copolymers. Structure and rheology. Macromolecules, 34, 8129-8137 (2001).

DOI: $10.1021 / \mathrm{ma} 0109440$

[29] Gopalan P., Zhang Y., Li X., Wiesner U., Ober C. K.: Liquid crystalline rod-coil block copolymers by stable free radical polymerization: Synthesis, morphology, and rheology. Macromolecules, 36, 3357-3364 (2003). DOI: $10.1021 / \mathrm{ma} 021573 \mathrm{u}$

[30] Almdal K., Koppi K. A., Bates F. S., Mortensen K.: Multiple ordered phases in a block copolymer melt. Macromolecules, 25, 1743-1751 (1992).

DOI: $10.1021 / \mathrm{ma} 00032 \mathrm{a} 019$

[31] Mok M. M., Pujari S., Burghardt W. R., Dettmer C. M., Nguyen S. T., Ellison C. J., Torkelson J. M.: Microphase separation and shear alignment of gradient copolymers: Melt rheology and small-angle X-ray scattering analysis. Macromolecules, 41, 5818-5829 (2008). DOI: $10.1021 / \mathrm{ma} 8009454$

[32] Bates F. S.: Block copolymers near the microphase separation transition. 2. Linear dynamic mechanical properties. Macromolecules, 17, 2607-2613 (1984).

DOI: $10.1021 / \mathrm{ma} 00142 \mathrm{a} 025$

[33] Rosedale J. H., Bates F. S.: Rheology of ordered and disordered symmetric poly(ethylenepropylene)-poly (ethylethylene) diblock copolymers. Macromolecules, 23, 2329-2338 (1990).

DOI: $10.1021 / \mathrm{ma} 00210 \mathrm{a} 032$

[34] Fredrickson G. H., Bates F. S.: Dynamics of block copolymers: Theory and experiment. Annual Review of Materials Science, 26, 501-550 (1996).

DOI: 10.1146/annurev.ms.26.080196.002441

[35] Kossuth M. B., Morse D. C., Bates F. S.: Viscoelastic behavior of cubic phases in block copolymer melts. Journal of Rheology, 43, 167-196 (1999).

DOI: $10.1122 / 1.550981$
[36] Han C. D., Baek D. M., Kim J. K., Ogawa T., Sakamoto N., Hashimoto T.: Effect of volume fraction on the order-disorder transition in low molecular weight polystyrene-block-polyisoprene copolymers. 1 . Orderdisorder transition temperature determined by rheological measurements. Macromolecules, 28, 50435062 (1995).

DOI: $10.1021 / \mathrm{ma} 00118 \mathrm{a} 038$

[37] Harris J. M.: Laboratory synthesis of polyethylene glycol derivatives. Polymer Reviews, 25, 325-373 (1985). DOI: $10.1080 / 07366578508081960$

[38] Dust J. M., Fang Z. H., Harris J. M.: Proton NMR characterization of poly(ethylene glycols) and derivatives. Macromolecules, 23, 3742-3746 (1990).

DOI: $10.1021 / \mathrm{ma} 00218 \mathrm{a} 005$

[39] Williams M. L., Landel R. F., Ferry J. D.: The temperature dependence of relaxation mechanisms in amorphous polymers and other glass-forming liquids. Journal of the American Chemical Society, 77, 3701-3707 (1955).

DOI: $10.1021 / \mathrm{ja} 01619 \mathrm{a} 008$

[40] Teoh R. L., Guice K. B., Loo Y-L.: Atom transfer radical copolymerization of hydroxyethyl methacrylate and dimethylaminoethyl methacrylate in polar solvents. Macromolecules, 39, 8609-8615 (2006).

DOI: $10.1021 / \mathrm{ma} 061650 \mathrm{~b}$

[41] Zhang J., Jiang L., Zhu L., Jane J-L., Mungara P.: Morphology and properties of soy protein and polylactide blends. Biomacromolecules, 7, 1551-1561 (2006). DOI: $10.1021 / \mathrm{bm} 050888 \mathrm{p}$

[42] Lu X., Hay J. N.: The effect of physical aging on the rates of cold crystallization of poly(ethylene terephthalate). Polymer, 41, 7427-7436 (2000).

DOI: $10.1016 / \mathrm{S} 0032-3861(00) 00092-6$

[43] O’Sickey M. J., Lawrey B. D., Wilkes G. L.: Structure-property relationships of poly(urethane-urea)s with ultralow monol content poly(propylene glycol) soft segments. III. Influence of mixed soft segments of ultralow monol poly(propylene glycol), poly(tetramethylene ether glycol), and tri(propylene glycol). Journal of Applied Polymer Science, 89, 3520-3529 (2003).

DOI: 10.1002/app.12520

[44] Xu Z., Niu Y., Yang L., Xie W., Li H., Gan Z., Wang Z.: Morphology, rheology and crystallization behavior of polylactide composites prepared through addition of five-armed star polylactide grafted multiwalled carbon nanotubes. Polymer, 51, 730-737 (2010). DOI: $10.1016 /$ j.polymer.2009.12.017

[45] Hillmyer M. A., Bates F. S.: Synthesis and characterization of model polyalkane-poly(ethylene oxide) block copolymers. Macromolecules, 29, 6994-7002 (1996). DOI: $10.1021 / \mathrm{ma} 960774 \mathrm{t}$

[46] Kelarakis A., Mai S-M., Booth C., Ryan A. J.: Can rheometry measure crystallization kinetics? A comparative study using block copolymers. Polymer, 46, 2739-2747 (2005).

DOI: $10.1016 /$ j.polymer.2005.01.068 